\title{
Potencial ornamental de especies nativas en el paisaje urbano del Valle Sagrado de los Incas, Cusco-Perú
}

\section{Ornamental potential of native species in the urban landscape of the Sacred Valley of the Incas, Cusco-Peru}

\section{Gloria Calatayud Hermoza , Isau Huamantupa-Chuquimaco", Alejandro Raya Tocre', Juan Cancio Chullo ${ }^{\prime}$, Fortunato Quispe $L^{1}$., Mario Huamantica ${ }^{\prime}$, Martin Llanos ${ }^{1}$, Mario Salas $^{1}$ \& Vittorino Sanchez ${ }^{1}$}

${ }^{1}$ Hotel Tambo del Inka, the Luxury Collection Resort \& spa - Urubamba. Email: calatayudhglor@gmail.com

${ }^{2}$ Centro Ecológico INKAMAZONIA, Valle de Kosñipata, via Cusco-RB Manú. Cusco, Perú.

\section{Introducción}

Las plantas han sido utilizadas por el hombre desde el inicio de las civilizaciones no solo como alimento, vestido o material de construcción, también, para decorar o adornar sus viviendas, palacios, espacios públicos, incluidos recintos religiosos o festivos en Chimal y Corona (2003). Las plantas ornamentales jugaron un rol importante en las culturas prehispánicas. Estas plantas se utilizaban también como medios de expresión religiosa, de agradecimiento, emblema de escudos, etc. (Chimal \& Corona, 2003).

El potencial ornamental de las plantas radica en sus valores estéticos (color, fragancia), utilitarios (durabilidad, resistencia), y económicos (Borys y Leszczyñska-Borys, 1992). Hablar de plantas ornamentales es tocar el tema de que valores tienen cada una de ellas que podría ser la forma natural (arquitectura), color de hojas, frutos, flores. El valor ornamental puede medirse en el tiempo, por ejemplo, la temporada de floración, defoliación, etc (Leszczyñska-Borys, 1990).
Muchas de las plantas se han utilizado para decorar construcciones: balcones, patios, ventanas, plazas, calles, etc. (Granziera, 2001; Leszczyñska-Borys, 1990 ; Borys y Leszczyñska-Borys, 1992; Rzedowski, 1995).

Actualmente son pocas las especies nativas que son utilizadas en la ornamentación de plazas, jardines de ciudades y hoteles en nuestro entorno. Las plantas ornamentales constituyen una herramienta fabulosa para restaurar y crear paisajes bellos. Tupayachi (2005), reporta 176 especies para la Cordillera del Vilcanota. De estas especies, 18 las hemos utilizado como especies ornamentales en los jardines del Hotel Tambo del Inka, the Luxury Collection Resort \& spa (HTI).

El objetivo de nuestro trabajo fue conocer, propagar y manejar especies nativas con potencial ornamental presentes en los alrededores del distrito de Urubamba y aportar en la adición de especies silvestres que creemos deben incorporarse en la ornamentación de jardines locales y regionales. Este objetivo fue parte de los parámetros en el diseño y la construcción del (HTI) (Fig. 1). 


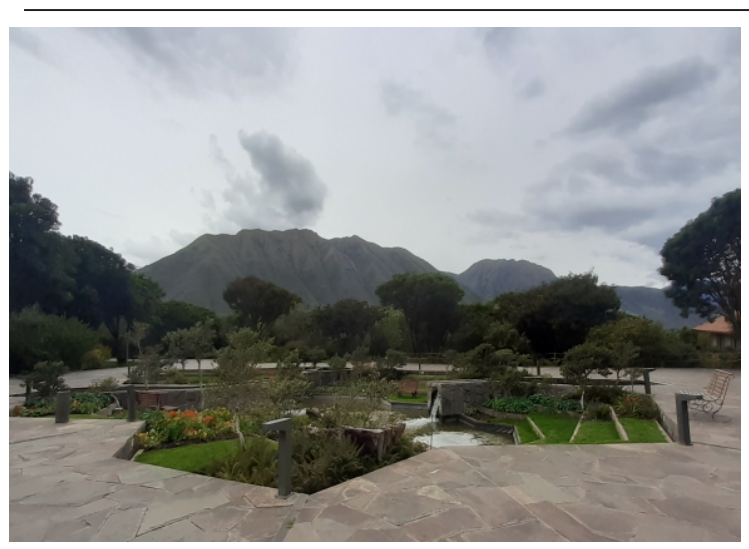

Figura 1. Jardines centrales del Hotel Tambo del Inka - Urubamba.

\section{Metodología}

Realizamos pruebas de aclimatación y manejo que se llevaron a cabo en las instalaciones del HTI ubicado en el distrito de Urubamba, región Cusco. El método que utilizamos fue:

1. Selección y recolección de especies con potencial ornamental. Para esta selección se tomó en cuenta los siguientes aspectos: Color y tamaño de las flores, aroma, arquitectura de la planta, duración y fechas del periodo de floración, tonos del color de las hojas, persistencia de las hojas, atracción a polinizadores y consumidores, mejoramiento de suelos. Esta selección es importante para adecuar las plantas de acuerdo a su apariencia y ubicación en los jardines.

2. Clasificamos a las plantas (50 especies entre árboles, arbustos, sub-arbustos y hierbas) considerando el tiempo de su desarrollo y su adaptación al nuevo ambiente que en primera instancia fue el vivero y luego los jardines, jardineras y macetas de interior y exterior. Se consideró las características ambientales y la la distribución de las especies (ver Cuadro $\mathrm{N}^{\circ} 1$ ).

3. Determinamos su uso potencial para jardines (como cubresuelo, de jardinera externa e interna, de maceta, de corte, como melífera, alimento de ves o insectos, protección o mejoramiento de suelos, tolerancia al manejo, sensible o no a ataque de plagas).

\section{Resultados}

Se catalogaron 50 especies de plantas ornamentales, pertenecientes a 28 familias y 37 géneros (Tab. 1).

Los jardines del HTI tienen una extensión de casi $6 \mathrm{Ha}$. A la fecha mantenemos un vivero, que nos sirve de herramienta importante para la conservación y recuperación de especies.

Las especies que tuvieron mejores resultados de manejo fueron Pulchea zamalloae, Baccharis boliviensis, Baccharis latifolia, Dodonaea viscosa, Salvia dombeyi, Fuchsia microphylla, Piper aduncum, Epidendrum secundum aff., Asplenium squamosum (Tab. 1).

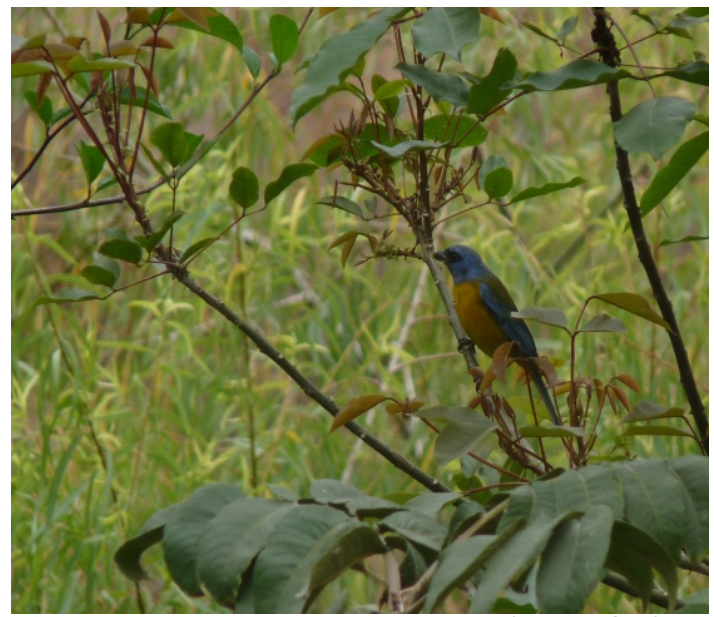

Figura 2. Pripaidea bonaherensis alimentándose de brotes foliares de Erythrina falcata. 
¿Qué retos tenemos? Seguir ejecutando actividades de culturización de plantas nativas con potencial ornamental, y en especial de las especies incluidas en alguna categoría de amenaza.

En el manejo de los jardines del HTI hemos considerado el mejoramiento de suelos, la resistencia a podas y el ataque de plagas. Las especies utilizadas han mostrado buena adaptación a podas en su mayoría. Sin embargo, Baccharis tricuneata (L. f.) Pers., y Lantana cámara L., vimos que son especies muy sensibles al ataque de la queresa (Lepidosaphes beckii Newman). Este insecto causó la muerte de muchos individuos. Por otro lado, Schinus molle L. y Myrcianthes oreophilla (Diels) McVaughes son especies sensibles al ataque de la queresa Ceroplastes floridensis Comstock. y Cuscuta grandiflora Kunth. El control de los mencionados insectos se realizó con tratamientos sugeridos por Narrea (2012). En el mejoramiento de suelos se utilizó tierra negra, tierra de hojas (topsoil) y compost $(3: 5: 2)$.

Presentamos una breve descripción de las especies más importantes por potencial de uso:

\section{Jardín}

Baccharis boliviensis y Baccharis latifolia: "Chilca”. Son arbustos que al realizar el trasplante se adaptan rápidamente a su nuevo medio, soportan sol intenso, podas y poco riego, no sufre de plagas. Son especies melíferas.

Dodonaea viscosa: “Chamán”. Es un arbusto que de igual manera soporta el traslado y trasplante. En su etapa de fructificación toda la planta se vuelve de aspecto rojizo. Puede usarse cerca de habitaciones pues repele el ataque de insectos y también no sufre de ataque de plagas, soporta sol intenso y poco riego.
Salvia dombeyi: "Salvia real". Es un sub arbusto apoyante que requiere de cuidado durante el inicio de su trasplante. Es una planta que debe plantarse bajo sombra, adaptable a la poda, pero no soporta la falta de riego.

Piper aduncum, "moco-moco". Arbusto que soporta el trasplante incluso en individuos de 2 $\mathrm{m}$ de alto. Requiere buen riego. Soporta poda y es adaptable a sol y sombra. No tiene problemas de ataque de insectos.

\section{Maceta}

Asplenium squamosum, "ullpo". Helecho herbáceo que se adapta a plantación para jardines, soporta el sol y plantación en maceta.

\section{Flores de Corte}

Hippeastrum machupijchense, Se adapta al corte y permanencia en floreros. El escapo puede durar entre 5 a 7 días. Epidendrum secundum aff., "Wiñay Wayna". Los racimos pueden durar en florero alrededor de 5 días.

\section{Frutos comestibles}

Schinus molle, "Molle". Los frutos de este árbol son consumidos por Turdus chiguaco .

Fuchsia boliviana, "Chimpu-chimpu”. Los frutos maduros son consumidos por gorriones y Turdus chiguaco. Sambucus nigra subsp. peruviana, "sauco". Frutos consumidos por Pheucticus auriventris,Turdus chiquaco.

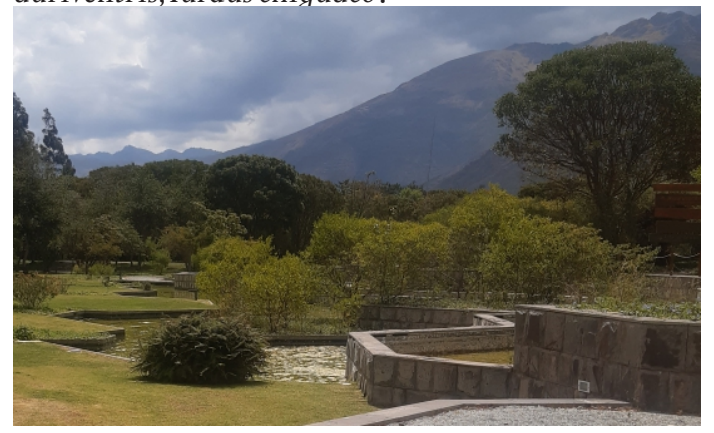

Figura 3. Se muestra en primer plano "Chamá” y "Pisonay" y al fondo "Kiswar" 
Tabla 1. Listado de especies nativas presentes en el distrito de Urubamba con potencial ornamental, primer grupo de importancia. A=Jardin, $\mathrm{B}=$ Maceta, $\mathrm{C}=$ Flor de corte, $\mathrm{D}=$ Frutos para Fauna, $\mathrm{E}=$ Melifera, $\mathrm{F}=$ Alimento de insectos (mariposas, abejas, avispas, etc) y $\mathrm{G}=\mathrm{Alimento}$ de aves

\begin{tabular}{|c|c|c|c|c|c|c|c|c|c|c|}
\hline \multirow[b]{2}{*}{ Familia } & \multirow[b]{2}{*}{ Especie } & \multirow[b]{2}{*}{ Nombre Quechua } & \multirow{2}{*}{$\begin{array}{c}\text { Especies } \\
\text { ornamentales } \\
\text { reportadas } \\
\text { como nuevas } \\
\text { como } \\
\text { ornamentales } \\
\end{array}$} & \multicolumn{3}{|c|}{ Potencial uso } & \multicolumn{4}{|c|}{$\begin{array}{l}\text { Otros usos útiles en } \\
\text { la jardinería }\end{array}$} \\
\hline & & & & $\mathbf{A}$ & B & $\mathrm{C}$ & D & $\mathbf{E}$ & $\mathbf{F}$ & G \\
\hline Anacardiaceae & Schinus molle L. & Molle & & $\mathbf{x}$ & $\mathbf{x}$ & & $\mathbf{x}$ & & & $\mathbf{x}$ \\
\hline Araliaceae & Oreopanax cuspidatus Harms & Maqui maqui & $\mathbf{x}$ & $\mathbf{x}$ & & & & & & \\
\hline Bignoniaceae & Tecoma sambusifolia Kunth & Huaranguay & & $\mathbf{x}$ & $\mathbf{x}$ & & & $\mathbf{x}$ & & $\mathbf{x}$ \\
\hline Bignoniaceae & Delostoma integrifolium D. Don & & & $\mathbf{x}$ & & & & & & $\mathbf{x}$ \\
\hline Buddlejaceae & Buddleja longifolia Kunth. & Kiswar & & $\mathbf{x}$ & & & & & & \\
\hline Buddlejaceae & Buddleja coriacea J. Rémy & Q'olle & & $\mathbf{x}$ & & & & & & \\
\hline Buxaceae & Styloceras laurifolium(Willd.) Kunth & Huamanquero & $\mathbf{x}$ & $\mathbf{x}$ & & & & & & \\
\hline Caprifoliaceae & Sambucus nigra subsp. peruviana(Kunth) Bolli & sauco & & $\mathbf{x}$ & & & $\mathbf{x}$ & $\mathbf{x}$ & & $\mathbf{x}$ \\
\hline Escalloniaceae & Escallonia resinosa (Ruiz \& Pav.) Pers. & Chachacomo & & $\mathbf{x}$ & & & & & & $\mathbf{x}$ \\
\hline Fabaceae & Erythrina falcata Benth. & Pisonay & & $\mathbf{x}$ & & & & $\mathbf{x}$ & & \\
\hline Fabaceae & Caesalpinia spinosa (Molina) Kuntze & Tara, taro & $\mathbf{x}$ & $\mathbf{x}$ & $\mathbf{x}$ & & & & & \\
\hline Grossulariaceae & Escallonia myrtilloides L. f. & Tasta & $\mathbf{x}$ & $\mathbf{x}$ & & & & & & \\
\hline Juglandaceae & Juglans neotropica Diels & Nogal & $\mathbf{x}$ & $\mathbf{x}$ & & & $\mathbf{x}$ & & & $\mathbf{x}$ \\
\hline Meliaceae & Cedrela angustifolia Sessé \& Moc. ex DC. & altura, siwis & & $\mathbf{x}$ & & & & & & $\mathbf{x}$ \\
\hline Myrtaceae & Myrcianthes oreophilla(Diels) McVaugh & Unca & & $\mathbf{x}$ & $\mathbf{x}$ & & & $\mathbf{x}$ & & \\
\hline Podocarpaceae & Podocarpus glomeratus D. Don & Intimpa & & $\mathbf{x}$ & & & & & & \\
\hline Rosaceae & Polylepis incana Kunth & Queuña & & $\mathbf{x}$ & $\mathbf{x}$ & & & & & \\
\hline Rosaceae & Polylepis pauta Hieron. & Queuña & & $\mathbf{x}$ & & & & & & \\
\hline Solanaceae & Solanum sp & Huanca huanca & $\mathbf{x}$ & $\mathbf{x}$ & & & & $\mathbf{x}$ & & \\
\hline Solanaceae & Nicotiana $s p$ & Tabaco falso & & $\mathbf{x}$ & & & & & & \\
\hline Verbenaceae & Citharexylum herrerae Mansf. & huayruro cusqueño & & $\mathbf{x}$ & $\mathbf{x}$ & & & $\mathbf{x}$ & & \\
\hline Verbenaceae & Citharexylum quercifolium Hayek. & Huayruro & & $\mathbf{x}$ & & & & & & \\
\hline Betulaceae & Alnus acuminata Kunth & Aliso & & & & & & & & \\
\hline Asteraceae & Baccharis tricuneataMansf. & Tayanka & $\mathbf{x}$ & $\mathbf{x}$ & & & & $\mathbf{x}$ & & \\
\hline Asteraceae & Baccharis boliviensis (Wedd.) Cabrera & Piqui piqui & $\mathbf{x}$ & $\mathbf{x}$ & & & & & & \\
\hline Asteraceae & Baccharis latifolia (Ruiz \& Pav.) Pers. & Chilca & $\mathbf{x}$ & $\mathbf{x}$ & & & & $\mathbf{x}$ & & \\
\hline Asteraceae & Baccharis latifolia cf. (Ruiz \& Pav.) Pers. & Chilca & $\mathbf{x}$ & $\mathbf{x}$ & & & & $\mathbf{x}$ & & \\
\hline Asteraceae & Pluchea zamalloae (Cabrera) H. R \& Cuatrec. & Chilca & $\mathbf{x}$ & $\mathbf{x}$ & & & & $\mathbf{x}$ & & \\
\hline Euphorbiaceae & $\begin{array}{l}\text { Dodonaea viscosa (L.) Jacq. } \\
\text { Senna birostris (Dombey ex Vogel) H.S. Irwin \& }\end{array}$ & chaman & & & & & & & & \\
\hline Fabaceae & $\begin{array}{l}\text { Barneby } \\
\text { Senna multiglandulosa(Jacq.) H.S. Irwin \& }\end{array}$ & Mutuy & & $\mathbf{x}$ & & & & & & $\mathbf{x}$ \\
\hline Fabaceae & Barneby & Mutuy & & $\mathbf{x}$ & & & & & & \\
\hline Fabaceae & Senna sp & Choclo de oro & & $\mathbf{x}$ & & & & & & \\
\hline Lamiaceae & Salvia dombeyi Epling & Nucchu real & $\mathbf{x}$ & $\mathbf{x}$ & & & & & $\mathbf{x}$ & \\
\hline Lamiaceae & Salviasp & Nucchu azul & $\mathbf{x}$ & $\mathbf{x}$ & $\mathbf{x}$ & & & & $\mathbf{x}$ & \\
\hline Onagraceae & Fuchsia boliviana Carrière & Fuxia & & $\mathbf{x}$ & $\mathbf{x}$ & & $\mathbf{x}$ & & $\mathbf{x}$ & $\mathbf{x}$ \\
\hline Onagraceae & Fuchsia microphylla Kunth & Fuxia enana & & $\mathbf{x}$ & $\mathbf{x}$ & & & & & $\mathbf{x}$ \\
\hline Onagraceae & Fuchsia sp & Fuxia & & & & & & & & $\mathbf{x}$ \\
\hline Piperaceae & Piper aduncum $\mathrm{L}$. & & $\mathbf{x}$ & $\mathbf{x}$ & & & & & & \\
\hline Polemoniaceae & Cantua buxifolia Juss. ex Lam & cantu, cantuta & & $\mathbf{x}$ & & & & & & $\mathbf{x}$ \\
\hline Solanaceae & Brugmansia candida Pers. & Campachu blanco & & $\mathbf{x}$ & & & & & & \\
\hline Solanaceae & Brugmansia sanguinea (Ruiz \& Pav.) D. Don & Campachu rojo & & $\mathbf{x}$ & & & & & & \\
\hline Solanaceae & Brugmansia suaveolens (ex Willd.). \& C. Presl & Campachu amarillo & & $\mathbf{x}$ & & & & $\mathbf{x}$ & & \\
\hline Solanaceae & Streptosolen jamesonii (Benth.) Miers & Caramelito & & $\mathbf{x}$ & $\mathbf{x}$ & & & & & \\
\hline Solanaceae & Lycianthes lycioides (L.) Hassl. & Tankar & $\mathbf{x}$ & $\mathbf{x}$ & & & & & & \\
\hline Verbenaceae & Lantana camara L. & Lauraymana & & $\mathbf{x}$ & $\mathbf{x}$ & & & & & $\mathbf{x}$ \\
\hline Verbenaceae & Duranta armata Moldenke & & & $\mathbf{x}$ & & & & & & $\mathbf{x}$ \\
\hline Crassulaceae & Echeveria $s p$ & & & $\mathrm{x}$ & $\mathrm{x}$ & & & & $\mathrm{x}$ & \\
\hline Amaryllidaceae & Hippeastrum machupijchense (Vargas) D. Hunt & & & $\mathrm{x}$ & $\mathrm{x}$ & $\mathrm{x}$ & & & $\mathrm{x}$ & \\
\hline Orchidaceae & Epidendrum secundum aff. Jacq. & & $\mathrm{x}$ & $\mathrm{x}$ & & $\mathrm{x}$ & & & $\mathrm{x}$ & \\
\hline Aspleniaceae & Asplenium squamosum L. & & $\mathrm{x}$ & $\mathrm{x}$ & $\mathrm{x}$ & & & & & \\
\hline
\end{tabular}




\section{Agradecimentos}

A Bruno Giordano, Pablo, Wilfredo Flores, Washington Gil y Rosario Chacón por su constante apoyo y facilidades prestadas. A Nikolas Aseshov por su identidad y cariño hacia las plantas nativas y estar al pendiente de nuestro trabajo. Al SERFOR por las facilidades en cuanto a los permisos de colección y centro de propagación de especies ornamentales de flora silvestre.

\section{Literatura citada}

Borys, M.W. \& Leszczcyñska-Borys, H. (1992). "Reflexiones sobre el Potencial Ornamental de Plantas Nativas de México". Serie: Manuales de Horticultura Ornamental, No. 7. Universidad Popular Autónoma del Estado.

Chimal, A. \& Corona,V.(2003). “Arbustos mexicanos con potencial ornamental”. En: Mejía, J. M y A. Espinosa (comps.). Plantas nativas de México con potencial ornamental. Universidad Autónoma de Chapingo, México.pp. 31-51.

Espejo, S. A. \& López, F. (1996). "Las Monocotiledóneas (Liliopsida) mexicanas con potencial ornamental, en: Mejía, J. M. y A. Espinosa (compiladores). Plantas Nativas de México con Potencial Ornamental. Universidad Autónoma de Chapingo, México. p. 20-26.

Espinosa, F. A., Gonzalales, R. H. \& Mejía, M. J.J. (2003). La comercialización de plantas en peligro de extinción, pp. 199-217. In: Plantas Nativas de México con Potencial Ornamental. Universidad Autónoma Chapingo. Chapingo, México. pp.199. 217.

Granziera, P. (2001). Concept of the Garden in Prehispanic Mexico. Garden History, Vol. 29, N² 2, pp. 185-213

Leszczyñska-Borys, H. \& Borys, M.W. (2003). "Plantas Ornamentales de la Sierra de Puebla". In: Mejía, M.J.M. y F.A. Espinosa (comps.). Plantas Nativas de México con Potencial Ornamental: Análisis y Perspectivas. Universidad Autónoma de Chapingo. pp. 132-145.

Narrea. C., M. (2012). Manejo integrado de plagas en el cultivo de cítricos. Guía técnica. Universidad Nacional Agraria La Molina - UNALM. Oficina Académica de Extensión y Proyección Social.
Rzedowski, J. (1995). "Aspectos de las Plantas Ornamentales Mexicanas". Revista Chapingo. Serie Horticultura. México. 1(3): 5-7.

Tupayachi, H. A. (2005). Flora de la Cordillera de Vilcanota. Arnaldoa 12 (1-2): 126-144. 